\title{
Optimal Sequences that Maximize the Information Theoretic Sum Capacity of Symbol Asynchronous CDMA Systems
}

\author{
Jie Luo, Sennur Ulukus, Anthony Ephremides \\ ECE Dept., University of Maryland \\ College Park, MD 20742 \\ Email: \{rockey, ulukus, tony\}@eng.umd.edu
}

\begin{abstract}
The optimal signature sequences that maximize the sum capacity of a direct sequence CDMA system are characterized in the general case of symbol delay profile and user power constraints. It is shown that the optimal sum capacity of the symbol asynchronous system equals that of the symbol synchronous system with the same user power constraints. With the optimal signature sequence set, the maximum sum capacity is achieved with white Gaussian input signals. The existence of the optimal signature sequence set is proved by the proposal of an explicit construction method for arbitrary user delay profiles and power constraints.
\end{abstract}

\section{INTRODUCTION}

D UE to the capability of offering high capacity, flexibility and security, DS-CDMA systems became popular in the early 1980's and have been studied extensively since then [1]. Among the research works on CDMA, there has been special interest in understanding the impact of signature sequences on the sum capacity of the system. Suppose that $K$ and $N$ are the number of users and the spreading gain of the system, respectively. For the symbol synchronous CDMA system, when the user powers are equal, [2] showed that the sum capacity is maximized by assigning an orthogonal signature sequence set to the users when $K \leq N$, and by assigning a Welch-BoundEquality (WBE) signature sequence set to the users when $K>N$. The general case of asymmetric user powers is solved in [3], where it is shown that the sum capacity is maximized by assigning orthogonal signatures to the oversized users (see equation (16) for a definition), and assigning a generalized WBE (GWBE) sequence set to the non-oversized users. Iterative methods are proposed in [3][4] to construct the optimal sequence sets. The signature sequence design problem for symbol asynchronous (but

This work was supported by National Aeronautics and Space Administration award No. NCC8-235, Collaborative Technology Alliance for Communication \& Networks sponsored by the U.S. Army Laboratory under Cooperative Agreement DAAD19-01-2-0011 and National Science Foundation grant ANI 02-05330. Any opinions findings, and conclusions or recommendations expressed in this material are those of the authors and do not necessarily reflect the views of the National Aeronautics and Space Administration or the Army Research Laboratory of the U.S. Government. chip synchronous) systems is studied recently in [5]. For a special case when the users are equal powered and when the matched filter receivers are used, the optimal sequence set that maximizes the user capacity has been found by minimizing the Total Squared Asynchronous Correlation (TSAC). An extension to the asymmetric user powers case was presented in [6]. It has been shown that the constraints on the optimal signature sequences in a symbol asynchronous system are stricter compared to those in a symbol synchronous system. We refer to the optimal signature sequence set obtained in [5] the Asynchronous WBE (AWBE) sequence set in this paper. Although simulations show that the AWBE sequences can be obtained by minimizing the TSAC, the existence of the AWBE sequence set under an arbitrary user delay profile was left as an open problem in [5][6].

In this paper, we study the signature sequence design problem from the sum information capacity point of view for asymmetric (i.e., unequal) user powers in a symbol asynchronous (but chip synchronous) CDMA system. We assume that the users are frame synchronous, and have a fixed deterministic delay profile. We show that the sum capacity of the symbol asynchronous system is upper bounded by that of the symbol synchronous system with the same power constraints. We derive the optimal signature sequences for the asynchronous system that achieve the sum capacity upper bound. Similar to the result in [3], we show that the optimal signature sequences for the symbol asynchronous system is obtained by assigning orthogonal signatures to the oversized users and assigning a Generalized AWBE (GAWBE) signature sequence set to the non-oversized users. We show that, when the users are equal-powered, the AWBE signature sequences given in [5] are indeed optimal. In addition, we present an explicit method to construct the optimal signature sequence set, given an arbitrary signal delay profile and general user power constraints. This solves the existence problem left open in [5]; in fact, it solves the existence problem in a more general setting of arbitrary powers.

\section{System Model}

We consider a DS-CDMA system where all $K$ users transmit to a single receiver. We assume that the user 
signals are chip synchronous; the chip waveform is identical for all users and is designed such that it can pass the bandwidth-limited channel with ignorable distortion. The processing gain is $N$.

Define $s_{k}=\left[\begin{array}{llll}s_{k 1} & s_{k 2} & \ldots & s_{k N}\end{array}\right]^{T}$ as the signature sequence of user $k$. Suppose the symbol delay of user $k$ is $c_{k}$ chips, where $c_{k}=0,1, \ldots, N-1$. We divide the signature sequence of user $k$ into two parts. The subvector $\tilde{\boldsymbol{s}}_{k}^{L}$ that contains the first $N-c_{k}$ chips is termed the left signature sequence, and the subvector $\tilde{\boldsymbol{s}}_{k}^{R}$ that contains the last $c_{k}$ chips is termed the right signature sequence, i.e.,

$$
\begin{aligned}
\tilde{\boldsymbol{s}}_{k}^{L} & =\left[s_{k 1}, s_{k 2}, \ldots, s_{k\left(N-c_{k}\right)}\right]^{T} \\
\tilde{\boldsymbol{s}}_{k}^{R} & =\left[s_{k\left(N-c_{k}+1\right)}, s_{k\left(N-c_{k}+2\right)}, \ldots, s_{k N}\right]^{T}
\end{aligned}
$$

In addition, define an $N \times 1$ vector $\boldsymbol{s}_{k}^{L}$ by padding 0 s to $\tilde{\boldsymbol{s}}_{k}^{L}$ from the top and define an $N \times 1$ vector $\boldsymbol{s}_{k}^{R}$ by padding 0s to $\tilde{\boldsymbol{s}}_{k}^{R}$ from the bottom.

$$
\boldsymbol{s}_{k}^{L}=[\overbrace{0, \ldots, 0}^{c_{k}}, \tilde{\boldsymbol{s}}_{k}^{L^{T}}]^{T}, \quad \boldsymbol{s}_{k}^{R}=[\tilde{\boldsymbol{s}}_{k}^{L^{R}}, \overbrace{0, \ldots, 0}^{N-c_{k} 0 \mathrm{~s}}]^{T}
$$

Consider the first $M$ symbol durations. The chip matched filter output of the receiver can be denoted by an $M N \times 1$ real-valued column vector $\boldsymbol{y}$, which satisfies the following system model

$$
\boldsymbol{y}=\sum_{k=1}^{K} \boldsymbol{S}_{k} \boldsymbol{x}_{k}+\boldsymbol{n}
$$

Here $\boldsymbol{y}=\left[\boldsymbol{y}(1)^{T}, \boldsymbol{y}(2)^{T}, \ldots, \boldsymbol{y}(M)^{T}\right]^{T}$, where $\boldsymbol{y}(m)$ is the chip-matched filter output vector of the $m^{\text {th }}$ symbol duration; $\boldsymbol{x}_{k}=\left[\begin{array}{llll}x_{k}(1) & x_{k}(2) & \ldots & x_{k}(M)\end{array}\right]^{T}$ is the source symbol vector of user $k ; \boldsymbol{n}$ is the Gaussian noise with zero mean and covariance matrix $E\left[\boldsymbol{n} \boldsymbol{n}^{T}\right]=\sigma^{2} \boldsymbol{I}_{M N}$, $\boldsymbol{I}_{M N}$ being the $M N \times M N$ identity matrix; and $\boldsymbol{S}_{k}$ is the signature matrix of user $k$, which is given by

$$
\boldsymbol{S}_{k}=\left[\begin{array}{cccc}
\boldsymbol{s}_{k}^{L} & 0 & \ddots & 0 \\
\boldsymbol{s}_{k}^{R} & \boldsymbol{s}_{k}^{L} & 0 & \ddots \\
0 & \ddots & \ddots & 0 \\
\ddots & 0 & \boldsymbol{s}_{k}^{R} & \boldsymbol{s}_{k}^{L}
\end{array}\right]
$$

We assume both the symbols and the signature sequences are real-valued. The average power of the normalized source signal of the $k^{t h}$ user is restricted to

$$
\operatorname{tr}\left(E\left[\boldsymbol{x}_{k} \boldsymbol{x}_{k}^{T}\right]\right) \leq M P_{k}
$$

where $P_{k}$ is the average power per symbol of user $k$. Given $J$ as an arbitrary group of users, the mutual information $I\left(\boldsymbol{x}_{k \in J} ; \boldsymbol{y} \mid \boldsymbol{x}_{k \notin J}\right)$ is given by [7]

$$
I\left(\boldsymbol{x}_{k \in J} ; \boldsymbol{y} \mid \boldsymbol{x}_{k \notin J}\right) \leq \frac{1}{2} \log _{2}\left[\left\lfloor\boldsymbol{I}_{M N}+\sum_{k \in J} \frac{\boldsymbol{S}_{k} E\left[\boldsymbol{x}_{k} \boldsymbol{x}_{k}^{T}\right] \boldsymbol{S}_{k}^{T}}{\sigma^{2}} \mid\right]\right.
$$

with equality if the signals of users $k \in J$ are Gaussian.

Assume that the user delay profile is known to both the transmitters and the receiver. The capacity region of the system is given by the convex closure, over independent random vectors $\boldsymbol{x}_{k}$ satisfying (5), of the union of the following heptagons.

$$
\begin{aligned}
& \lim _{M \rightarrow \infty} \bigcap_{J \subset\{1,2, \ldots, K\}}\left\{\left(R_{1}, \ldots, R_{K}\right):\right. \\
& \left.0 \leq \sum_{k \in J} R_{k} \leq \frac{1}{M} I\left(\boldsymbol{x}_{k \in J} ; \boldsymbol{y} \mid \boldsymbol{x}_{k \notin J}\right)\right\}
\end{aligned}
$$

Suppose the signature sequences are given. In the situation when user signals are symbol synchronous, it is shown in [7] that the rate constraints are maximized if the source signals have white power spectra, i.e., $E\left[\boldsymbol{x}_{k} \boldsymbol{x}_{k}^{T}\right]=P_{k} \boldsymbol{I}_{M}$, $\forall k$. However, in the symbol asynchronous case, in general, there is no unique power spectrum that can maximize the rate upper bounds in (7) simultaneously [7].

In the literature, the sum capacity of the rate region, defined as $\max \left(\sum_{k=1}^{K} R_{k}\right)$, has been of particular interest since it is a single number that represents the overall capacity limit of the system. Combining equation (6) and (7), the sum capacity per symbol of the system satisfies

$$
\begin{aligned}
C_{\text {sum }} \leq & \lim _{M \rightarrow \infty} \underset{\operatorname{tr}\left(E\left[\boldsymbol{x}_{k} \boldsymbol{x}_{k}^{T}\right]\right) \leq M P_{k}}{\max } \\
& \frac{1}{2 M} \log _{2}\left[\left|\boldsymbol{I}_{M N}+\sum_{k=1}^{K} \frac{\boldsymbol{S}_{k} E\left[\boldsymbol{x}_{k} \boldsymbol{x}_{k}^{T}\right] \boldsymbol{S}_{k}^{T}}{\sigma^{2}}\right|\right]
\end{aligned}
$$

where equality holds when the input signals are stationary Gaussian.

\section{Upper Bound on The Sum CApacity}

Suppose that the power of each symbol of the user is upper bounded. The following Theorem shows that, when calculating the channel capacity, we can replace the signature matrices by their corresponding block circulant versions.

Theorem 1: Suppose the eigenvalues of $E\left[\boldsymbol{x}_{k} \boldsymbol{x}_{k}^{T}\right]$ are uniformly bounded, which is true for most of the practical signals of interest. Define the block circulant signature matrix $\hat{\boldsymbol{S}}_{k}$ of user $k$ by adding the $N \times 1$ column vector $\boldsymbol{s}_{k}^{R}$ to the up-right corner of $\boldsymbol{S}_{k}$ in (4). Then for any set of users $J$, the two matrices $\sum_{k \in J} \hat{\boldsymbol{S}}_{k} E\left[\boldsymbol{x}_{k} \boldsymbol{x}_{k}^{T}\right] \hat{\boldsymbol{S}}_{k}^{T}$ and $\sum_{k \in J} \boldsymbol{S}_{k} E\left[\boldsymbol{x}_{k} \boldsymbol{x}_{k}^{T}\right] \boldsymbol{S}_{k}^{T}$ are asymptotically equivalent (see the definition in [8]).

The proof of is provided in [10].

According to Theorem 1 and [8, Theorem 2.3], the sum capacity in (8) can be written as

$$
\begin{aligned}
C_{\text {sum }} \leq & \lim _{M \rightarrow \infty} \underset{\operatorname{tr}\left(E\left[\boldsymbol{x}_{k} \boldsymbol{x}_{k}^{T}\right]\right) \leq M P_{k}}{ } \\
& \frac{1}{2 M} \log _{2}\left[\left|\boldsymbol{I}_{M N}+\sum_{k=1}^{K} \frac{\hat{\boldsymbol{S}}_{k} E\left[\boldsymbol{x}_{k} \boldsymbol{x}_{k}^{T}\right] \hat{\boldsymbol{S}}_{k}^{T}}{\sigma^{2}}\right|\right]
\end{aligned}
$$

Note that all circulant matrices of the same dimension have identical eigenvector set. Define the $M \times 1$ vector $\boldsymbol{q}_{m}$ 
and the $M \times M$ Fourier transform matrix $\boldsymbol{Q}_{M}$ as

$$
\begin{aligned}
& \boldsymbol{q}_{m}=\left[\begin{array}{llll}
1 & e^{j \frac{2 \pi(m-1)}{M}} & \ldots & e^{j \frac{2 \pi(m-1)(M-1)}{M}}
\end{array}\right]^{H} \\
& \boldsymbol{Q}_{M}=\frac{1}{\sqrt{M}}\left[\begin{array}{llll}
\boldsymbol{q}_{1} & \boldsymbol{q}_{2} & \ldots & \boldsymbol{q}_{M}
\end{array}\right]
\end{aligned}
$$

Denote the $m^{\text {th }}$ component of vector $\boldsymbol{q}_{n}$ by $q_{n m}$. According to [8], we can decompose the block circulant matrix $\hat{\boldsymbol{S}}_{k}$ as

$$
\hat{\boldsymbol{S}}_{k}=\left[\begin{array}{ccc}
q_{11} \boldsymbol{I}_{N} & \ddots & q_{M 1} \boldsymbol{I}_{N} \\
\ddots & \ddots & \ddots \\
q_{1 M} \boldsymbol{I}_{N} & \ddots & q_{M M} \boldsymbol{I}_{N}
\end{array}\right] \boldsymbol{\Phi}_{k}^{*} \boldsymbol{Q}_{M}^{H}
$$

where $\boldsymbol{\Phi}_{k}$ is a block diagonal matrix, defined as,

$$
\boldsymbol{\Phi}_{k}=\left[\begin{array}{cccc}
\phi_{k 1} & 0 & \ddots & 0 \\
0 & \phi_{k 2} & \ddots & \ddots \\
\ddots & \ddots & \ddots & \ddots \\
0 & \ddots & 0 & \phi_{k M}
\end{array}\right]
$$

and $\phi_{k m}$ in equation (12) is an $N \times 1$ column vector given by

$$
\phi_{m}=\boldsymbol{s}_{k}^{L}+\boldsymbol{s}_{k}^{R} e^{-j \frac{2 \pi(m-1)}{M}}
$$

Substituting (11) into (9), we get

$$
\begin{aligned}
& \left|\boldsymbol{I}_{M N}+\sum_{k=1}^{K} \frac{\hat{\boldsymbol{S}}_{k} E\left[\boldsymbol{x}_{k} \boldsymbol{x}_{k}^{T}\right] \hat{\boldsymbol{S}}_{k}^{T}}{\sigma^{2}}\right| \\
& =\left|\boldsymbol{I}_{M N}+\sum_{k=1}^{K} \frac{\boldsymbol{\Phi}_{k}^{*} \boldsymbol{Q}_{M}^{H} E\left[\boldsymbol{x}_{k} \boldsymbol{x}_{k}^{T}\right] \boldsymbol{Q}_{M} \boldsymbol{\Phi}_{k}^{T}}{\sigma^{2}}\right| \\
& \leq\left|\boldsymbol{I}_{M N}+\sum_{k=1}^{K} \frac{\boldsymbol{\Phi}_{k}^{*} \boldsymbol{P}_{k} \boldsymbol{\Phi}_{k}^{T}}{\sigma^{2}}\right|
\end{aligned}
$$

where $\boldsymbol{P}_{k}$ is an $M \times M$ diagonal matrix, whose diagonal entries are equal to those of $\boldsymbol{Q}_{M}^{H} E\left[\boldsymbol{x}_{k} \boldsymbol{x}_{k}^{T}\right] \boldsymbol{Q}_{M}$. The last inequality in (14) is due to the generalized Hadamard inequality [7].

Denote the $m^{\text {th }}$ diagonal entry of $\boldsymbol{P}_{k}$ by $p_{k m}$. Since equation (14) holds with equality when $\boldsymbol{Q}_{M}^{H} E\left[\boldsymbol{x}_{k} \boldsymbol{x}_{k}^{T}\right] \boldsymbol{Q}_{M}$ is diagonal, substituting (14) into (9), we obtain

$$
\begin{aligned}
& C_{\text {sum }} \leq \lim _{M \rightarrow \infty} \max _{m=1}^{M} p_{k m} \leq M P_{k} \\
& \frac{1}{2 M} \sum_{m=1}^{M} \log _{2}\left[\left|\boldsymbol{I}_{N}+\sum_{k=1}^{K} \frac{\boldsymbol{\phi}_{k m}^{*} p_{k m} \boldsymbol{\phi}_{k m}^{T}}{\sigma^{2}}\right|\right]
\end{aligned}
$$

When the signature sequences of the users are given, the sum capacity can be found by solving (15) with an iterative water filling algorithm [7]. It is easily seen that, given an arbitrary signature set and user delay profile, the sum capacity is usually not achieved by input signals with white spectra (i.e., $\boldsymbol{P}_{k}=P_{k} \boldsymbol{I}_{M}$ ).

In the following Theorem, we give an upper bound on the sum capacity. The upper bound has the feature that it is not a function of the signature sequences of the users.
Theorem 2: Similar to [3], define user $k$ as oversized if

$$
P_{k}>\frac{\sum_{j=1}^{K} P_{j} 1_{P_{k}>P_{j}}}{N-\sum_{j=1}^{K} 1_{P_{k} \leq P_{j}}}
$$

Denote the set of the oversized users as $\mathcal{K}$. The sum capacity is upper bounded by

$$
\begin{aligned}
C_{\text {sum }} \leq & \frac{N-|\mathcal{K}|}{2} \log _{2}\left(1+\frac{\sum_{k \notin \mathcal{K}} P_{k}}{(N-|\mathcal{K}|) \sigma^{2}}\right) \\
& +\frac{1}{2} \sum_{k \in \mathcal{K}} \log _{2}\left(1+\frac{P_{k}}{\sigma^{2}}\right)
\end{aligned}
$$

This upper bound is equal to the optimal sum capacity of the symbol synchronous system, as given in [3].

The proof is given in [10].

\section{Optimal Signature Sequences}

Although the upper bound given in (17) may not be achieved with an arbitrary signature sequence set, we show in this section that, it is indeed achievable when the signature sequences are optimally designed, given any arbitrary user delay profile and average power constraints.

\section{A. The Necessary and Sufficient Condition}

The following Lemma shows the connection between the Frobenius norm, denoted by $\|\cdot\|_{F}$, and the eigenvalues of a symmetric matrix $\boldsymbol{A}$.

Lemma 1: Suppose $\boldsymbol{A}$ is an $N \times N$ positive definite matrix that satisfies $\boldsymbol{A}^{H}=\boldsymbol{A}$. Suppose $\tilde{N} \leq N$ and $\lambda_{1}, \ldots, \lambda_{\tilde{N}}$ are all the non-zero eigenvalues of $\boldsymbol{A}$. If $\boldsymbol{A}$ is constrained such that $\operatorname{tr}(\boldsymbol{A})=P$, then $\|\boldsymbol{A}\|_{F}^{2} \geq \frac{P^{2}}{\tilde{N}}$ with equality if and only if $\lambda_{1}=\lambda_{2}=\ldots=\lambda_{\tilde{N}}=\frac{P}{\tilde{N}}$.

The proof is given in [10].

With the help of Lemma 1, we are now ready to present the necessary condition on the optimal signature sequence set, which guarantees that the sum capacity achieves the upper bound given in (17). Somewhat surprisingly, the condition becomes sufficient if the input signals have white spectra.

Theorem 3: If $\mathcal{K}$ is the set of oversized users, given the user delay profile and the power constraints, if the sum capacity upper bound of (17) is achieved with equality, then the input signals are Gaussian and the following three conditions hold.

- Condition 1: For all $k \in \mathcal{K}$ and $j \neq k$

$$
\begin{aligned}
& \boldsymbol{s}_{k}^{L^{T}} \boldsymbol{s}_{j}^{L}+\boldsymbol{s}_{k}^{R^{T}} \boldsymbol{s}_{j}^{R}=0 \\
& \boldsymbol{s}_{k}^{L^{T}} \boldsymbol{s}_{j}^{R}=0 \text { and } \boldsymbol{s}_{k}^{R^{T}} \boldsymbol{s}_{j}^{L}=0
\end{aligned}
$$

The input signal of user $k \in \mathcal{K}$ has white power spectrum.

- Condition 2:

$$
\sum_{k \notin \mathcal{K}} P_{k} \boldsymbol{s}_{k}^{L} \boldsymbol{s}_{k}^{R^{T}}=0
$$


- Condition 3:

$$
\left\|\sum_{k \notin \mathcal{K}} P_{k}\left(\boldsymbol{s}_{k}^{L} \boldsymbol{s}_{k}^{L^{T}}+\boldsymbol{s}_{k}^{R} \boldsymbol{s}_{k}^{R^{T}}\right)\right\|_{F}^{2}=\frac{\left(\sum_{k \notin \mathcal{K}} P_{k}\right)^{2}}{N-|\mathcal{K}|}
$$

Furthermore, given the user delay profile and the average power constraints, if equations (18), (19), (20) hold, and the user signals are white Gaussian, i.e., $E\left[\boldsymbol{x}_{k} \boldsymbol{x}_{k}^{T}\right]=$ $P_{k} \boldsymbol{I}_{M}, \forall k$, then the sum capacity of the system achieves the upper bound given in (17).

The proof is presented in [10].

To be consistent with [3], we term the signature sequence set of the non-oversized users that satisfy conditions (18), (19) and (20) the generalized asynchronous Welch-Bound-Equality (GAWBE) sequences.

Note that in the equal user power case, with condition (19), $\left\|P_{k} \sum_{k \notin K}\left(s_{k}^{L} \boldsymbol{s}_{k}^{L^{T}}+\boldsymbol{s}_{k}^{R} \boldsymbol{s}_{k}^{R^{T}}\right)\right\|_{F}^{2}$ is proportional to the TSAC of the system, defined in [5], which is also the TSC when the user signals are symbol synchronous. Under the constraint of (19), Lemma 1 implies that minimizing the TSC or the TSAC results in the optimal signature sequences.

\section{B. Construction of the Optimal Signature Sequences}

Assume that the input signals have white spectra. Although Theorem 3 gives the necessary and sufficient condition for the sum capacity to reach its upper bound, it can be seen that the conditions for the symbol asynchronous system are much stricter than the symbol synchronous case in general. Hence, given an arbitrary power constraint set and a user delay profile, it is natural to ask whether such optimal signature sequence set always exists. In this section, we provide an explicit algorithm to construct the optimal signature sequence set that satisfies all three conditions in Theorem 3. The construction algorithm guarantees that the upper bound in equation (17) is always achieved with i.i.d. Gaussian inputs.

First, we consider the situation when there is no oversized users, i.e., the power of all the users satisfy

$$
P_{k} \leq \frac{\sum_{j=1}^{K} P_{j}}{N}, \quad \forall k
$$

Our purpose is to construct a signature sequence set such that $\boldsymbol{s}_{k}^{R}=0$ for all $k$. Although this guarantees that the requirement in (19) is met, unfortunately, meeting the requirement in (20) with such a strict constraint is not always possible for a general user delay profile. We use the time labeling idea presented in [9], that enables us to work with other $N-1$ equivalent delay profiles, even when the user delay profile is given. This significantly increases our freedom in the signature design. We will show that, among all the $N$ equivalent user delay profiles, there always exists one that allows us to construct an optimal signature sequence set that simultaneously satisfies $\boldsymbol{s}_{k}^{R}=0, \forall k$ and condition in (20).

Arrange the users in increasing order of their delays, i.e., $c_{1} \leq c_{2} \leq \ldots \leq c_{K}$. Since all $c_{k}$ take values in
[0,N-1], we can divide the users into $N$ groups, denoted by $G_{1}, G_{2}, \ldots, G_{N}$, such that the users in the same group are symbol synchronous. Specifically, the delay of the users satisfy

$$
c_{k}=i-1, \quad \text { if } k \in G_{i}
$$

The aggregate signal power in group $G_{j}$ is denoted by $P_{G_{j}}=\sum_{k \in G_{j}} P_{k}$. It is possible that some of the groups are empty, depending on the user delay profile.

Note that (22) is based on the assumption that the users in group $G_{1}$ have zero delays. Suppose we pick an arbitrary time labeling and define it as $T_{1}=\left[G_{1}, G_{2}, \ldots, G_{N}\right]$. Now, without changing the physical signals, if we change the time labels and define the users in group $G_{N}$ as the zero-delayed users, we can reorder the groups chronologically according to the new time labels and get another equivalent group ordering, $T_{2}=\left[G_{N}, G_{1}, G_{2} \ldots, G_{N-1}\right]$. Overall, there are $N$ different time labelings, and each time labeling is uniquely determined by the corresponding chronological order of the group. We say that time labeling $T_{2}$ is obtained from $T_{1}$ through a backward rotation.

The following Theorem shows that among the $N$ different time labelings, there is one time labeling that possesses a special property, which is the key feature that ensures the existence of the optimal signature sequence set.

Theorem 4: Assume that there are no oversized users. Among all the time labelings, there exists one time labeling $\hat{T}=\left[\hat{G}_{1}, \hat{G}_{2}, \ldots, \hat{G}_{N}\right]$, such that $\forall 1 \leq j \leq N$, the signal powers satisfy

$$
\sum_{j=1}^{g} P_{\hat{G}_{j}} \geq \frac{g \sum_{k=1}^{K} P_{k}}{N}
$$

The proof is presented in [10].

Now, consider the time labeling $\hat{T}$, and arrange the users and the groups in the chronological order of their symbol delays. For notational simplicity, we still term these groups $G_{1}, G_{2}, \ldots, G_{N}$. Consider the signature ma$\operatorname{trix} \tilde{\boldsymbol{S}}=\left[\begin{array}{lll}\tilde{\boldsymbol{s}}_{1} & \ldots & \tilde{\boldsymbol{s}}_{K}\end{array}\right]$ where $\tilde{\boldsymbol{s}}_{k}=\left[\tilde{\boldsymbol{s}}_{k}^{R^{T}}, \tilde{\boldsymbol{s}}_{k}^{L^{T}}\right]^{T}$ is constructed by stacking the right signature sequence and the left signature sequence of user $k$ together. Here the left signature sequence $\tilde{\boldsymbol{s}}_{k}^{L}$ and the right signature sequence $\tilde{\boldsymbol{s}}_{k}^{R}$ are defined as in (1) according to the symbol delay $c_{k}$ of user $k$ in time labeling $\hat{T}$. Next, we show that one can always construct a GAWBE sequence set, such that all the components of the right signature sequences are 0s.

\section{Construction of the GAWBE sequence set}

- Step 1: Initialize $\boldsymbol{h}_{0}$ as a $K \times 1$ column vector whose first $N$ components have identical values of $\frac{\sum_{k=1}^{K} P_{k}}{N}$ and other components are 0, i.e.,

$$
\boldsymbol{h}_{0}=\left[\frac{\sum_{k=1}^{K} P_{k}}{N}, \ldots, \frac{\sum_{k=1}^{K} P_{k}}{N}, 0, \ldots, 0\right]^{T}
$$

Initialize matrix $\boldsymbol{H}_{0}=\operatorname{diag}\left(\boldsymbol{h}_{0}\right)$. Let $i=1$.

- Step 2: Construct a $K \times 1$ column vector $\boldsymbol{h}_{i}$ such that the following properties are satisfied

- Property 1: All the components of $\boldsymbol{h}_{i}$ are nonnegative. 
- Property 2: The first $N-i$ components of $\boldsymbol{h}_{i}$ have value $\frac{\sum_{k=1}^{K} P_{k}}{N}$.

- Property 3: The last $\sum_{j=N-i+1}^{N}\left|G_{j}\right|$ components of $\boldsymbol{h}_{i}$ are the powers of users in groups $G_{N-i+1}, \ldots, G_{N}$, respectively, in the same order.

- Property 4: The components located between the $N-i^{t h}$ and the $\sum_{j=1}^{N-i}\left|G_{j}\right|+1^{s t}$ components satisfy

$$
\left[\boldsymbol{h}_{i}\right]_{j} \leq\left[\boldsymbol{h}_{i-1}\right]_{j}, \quad \forall N-i<j \leq \sum_{j=1}^{N-i}\left|G_{j}\right|
$$

If we denote the powers of users in group $G_{i}$ as $P_{1}^{\left(G_{i}\right)}, \ldots, P_{\left|G_{i}\right|}^{\left(G_{i}\right)}, \boldsymbol{h}_{i}$ can be described as

$$
\begin{aligned}
\boldsymbol{h}_{i}= & {[\overbrace{\frac{\sum_{k=1}^{K} P_{k}}{N}, \ldots, \frac{\sum_{k=1}^{K} P_{k}}{N}}^{N-i \text { items }}, ?, \ldots, ?,} \\
& \left.P_{1}^{\left(G_{N-i+1}\right)}, P_{2}^{\left(G_{N-i+1}\right)}, \ldots, P_{\left|G_{N}\right|}^{\left(G_{N}\right)}\right]^{T}
\end{aligned}
$$

where the components marked with "?" can be arbitrary so long as they satisfy the inequality in (25). Note that such an $\boldsymbol{h}_{i}$ vector always exists since from (23) we have, $\sum_{j=1}^{N-i} P_{G_{j}} \geq \frac{(N-i) \sum_{k=1}^{K} P_{k}}{\sum^{N}}$ and $\sum_{j=1}^{N} P_{G_{j}}=\sum_{k=1}^{K} P_{k}$, which yields $\sum_{j=N-i+1}^{N} P_{G_{j}} \leq$ $\frac{i \sum_{k=1}^{K} P_{k}}{N}$.

Since $\boldsymbol{h}_{i-1}$ and $\boldsymbol{h}_{i}$ satisfy the inequality (25), by following the method in section IV of [3], we can construct a unitary matrix $\boldsymbol{U}_{N-i+1}$, such that the diagonal components of $\boldsymbol{U}_{N-i+1}^{T} \boldsymbol{H}_{i-1} \boldsymbol{U}_{N-i+1}$ are exactly the components of $\boldsymbol{h}_{i}$, in the same order. We define $\boldsymbol{H}_{i}=\boldsymbol{U}_{N-i+1}^{T} \boldsymbol{H}_{i-1} \boldsymbol{U}_{N-i+1}$.

- Step 3: If $i<N$, let $i=i+1$ and goto Step 2 . Otherwise, goto Step 4.

- Step 4: Define $\boldsymbol{U}=\boldsymbol{U}_{N} \boldsymbol{U}_{N-1} \ldots \boldsymbol{U}_{1}$, and define the matrix that contains the first $N$ rows of $\boldsymbol{U}$ as $\boldsymbol{V}$. We can then construct the signature sequence matrix as

$$
\tilde{\boldsymbol{S}}=\sqrt{\frac{\sum_{k=1}^{K} P_{k}}{N}} \boldsymbol{V} \operatorname{diag}\left(\frac{1}{\sqrt{P_{1}}}, \ldots, \frac{1}{\sqrt{P_{K}}}\right)
$$

Theorem 5: The GAWBE signature sequences constructed via the above procedure satisfies the conditions (19) and (20) in Theorem 3, for any time labeling.

The proof is presented in [10].

Compared with the optimal signature sequence construction in the symbol synchronous case [3], the key steps are that, first, we operate on the diagonal components of the $\boldsymbol{H}_{i}$ matrices in a specific order, and second, that the time labeling $\hat{T}$ satisfies the property shown in (23).

In the general case where there are $|\mathcal{K}|$ oversized users, we arrange the users such that users $1, \ldots, K-|\mathcal{K}|$ are non-oversized and users $K-|\mathcal{K}|+1, \ldots, K$ are oversized.
Now, we partition the signature sequence matrix $\tilde{\boldsymbol{S}}$ as

$$
\tilde{\boldsymbol{S}}=\left[\begin{array}{cc}
\tilde{\boldsymbol{S}}_{K-|\mathcal{K}|} & \mathbf{0} \\
\mathbf{0} & \boldsymbol{I}_{|\mathcal{K}|}
\end{array}\right]
$$

The signature sequence of user $k>K-|\mathcal{K}|$ is designed as $\tilde{\boldsymbol{s}}_{k}=\boldsymbol{e}_{k}$, where $\boldsymbol{e}_{k}$ is the column vector whose $k^{t h}$ component is 1 and all other components are 0 . The signature sequences of the non-oversized users are designed such that $\tilde{s}_{k j}=0, \forall k \leq K-|\mathcal{K}|$ and $j>N-|\mathcal{K}|$. Consequently, (18) is satisfied irrespective of the values of the components in the upper-left block matrix $\tilde{\boldsymbol{S}}_{K-|\mathcal{K}|}$.

Now consider the subblock matrix $\tilde{\boldsymbol{S}}_{K-|\mathcal{K}|}$ with dimension $(N-|\mathcal{K}|) \times(K-|\mathcal{K}|)$ in (28). The signature sequence design of $\tilde{\boldsymbol{S}}_{K-|\mathcal{K}|}$ is equivalent to that of a $K-|\mathcal{K}|$ user system with spreading factor of $N-|\mathcal{K}|$, where no oversized users are present. Therefore, we can assign the GAWBE sequence set for $\tilde{\boldsymbol{S}}_{K-|\mathcal{K}|}$, which completes the optimal sequence construction of the $K$ user system.

\section{Conclusion}

The optimal signature sequences that maximize the sum capacity of a symbol asynchronous CDMA system are characterized in this paper. By assigning orthogonal signature sequences to the oversized users, and assigning a GAWBE signature sequence set to the non-oversized users, asynchronous system achieves the same sum capacity as the synchronous system with the same user power constraints. An explicit method is provided for the construction of the optimal signature sequence set. This resolves the existence of the optimal signature sequences for arbitrary user delay profiles, and average power constraints.

\section{REFERENCES}

[1] S. Verdu, Multiuser Detection, Cambridge University Press, New York, 1998.

[2] M. Rupf and J. Massey, Optimum Sequence Multisets for Synchronous Code-Division Multiple-Access Channels, IEEE Trans. Info. Theory, Vol. 40, No. 4, pp. 1261-1266, July 1994.

[3] P. Viswanath and V. Anantharam, Optimum Sequences and Sum Capacity of Synchronous CDMA Systems, IEEE Trans. Info. Theory, Vol. 45, No. 6, pp. 1984-1991, Sep. 1999.

[4] S. Ulukus and R. Yates, Iterative Construction of Optimum Signature Sequence Sets in Synchronous CDMA Systems, IEEE Trans. Info. Theory, Vol. 47, No. 5, pp. 1989-1998, July 2001.

[5] S. Ulukus and R. Yates, User Capacity of Asynchronous CDMA Systems with Matched Filter Receivers and Optimum Signature Sequences, IEEE Trans. on Info. Theory, Vol. 50, No. 5, pp. 903909, May 2004.

[6] S. Stanczak and H. Boche, Optimal Signature Sequences for Asynchronous CDMA Systems with Fixed Signal Delays, Proc. 5th International Symposium on Wireless Personal Multimedia Communications, Honolulu, Hawaii, pp. 27-30, Oct. 2002.

[7] S. Verdu, The Capacity Region of the Symbol-Asynchronous Gaussian Multiple-Access Channel, IEEE Trans. Info. Theory, Vol. IT-35, No. 4, pp. 733-751, July 1989.

[8] R. Gray, On the Asymptotic Eigenvalue Distribution of Toeplitz Matrices, IEEE Trans. Info. Theory, Vol. IT-18, No. 6, pp. 725730, Nov. 1972.

[9] J. Luo, K. Pattipati, P. Willett and F. Hasegawa, Optimal User Ordering and Time Labeling for Decision Feedback Detection in Asynchronous CDMA, IEEE Trans. Commun., Vol. 51, No. 11, pp. 1754-1757, Nov. 2003.

[10] J. Luo, S. Ulukus and A. Ephremides, Optimal Sequences and Sum Capacity of Symbol Asynchronous CDMA Systems, submitted to IEEE Trans. Info. Theory, May 2004. 